\title{
AN Adaptive Neuro Fuzzy Inference System Control For A Bearingless Induction Motor Based On A Disturbance Observer
}

\author{
Panchagnula Sai Harish, S Sridhar
}

\begin{abstract}
There is some poor performance regarding controlling capacity of the bearing-less induction motor (BIM) when there are deviations in the parameters, outer disturbances and changes in the loads. So to solve this issue design of an adaptive exponential sliding-mode (AESM) controller and an observer for extended SM disturbance for finding system disturbance variables while operating are done. This adaptive exponential control is explained by combining order one norm and switching function law into regular control strategy. We can adjust the conjuction speed time adaptively as per variation of the SM switch surface and the system status. The controller used in this control strategy is Adaptive Neuro-Fuzzy Inference System (ANFIS). The observer used senses the speed and outer disturbances of the bearing-less induction motor. As feed forward contribution for system speed, the response of DSMO is utilized. The disturbance in the motor can be reduced by adjusting error in the speed by this feedback speed. From simulation output it can be seen that proposed system with ANFIS control strategy has good strength to control disturbances and to find the uncertain disturbances accurately. Hence the controlling capacity of the bearing-less induction motor (BIM) when there are deviations can be improved by using this proposed system.
\end{abstract}

Keywords : Adaptive exponential sliding mode (AESM), Bearing-less Induction Motor (BIM), Disturbance sliding mode observer (DSMO), Sliding mode (SM)

\section{INTRODUCTION}

The bearing-less induction machine (BIM) has the elements of levitation and spin. Based on its particular capacity, the BIM can keep running in a unique situation without grinding and wear. It is used in artificious heart, aircraft and space navigations, and computer drive, etc [1-2]. Simultaneously, the BIM incorporates the two arrangements in the stator turns and breaks the attractive field circulation of the traditional engines to understand the capacity of levitation and turn. So, this results in high mechanical quality and elementary structure of BIM. Yet, the BIM is a convoluted framework that has qualities of nonlinear, multi-variable and solid coupling, the prerequisite of controller ought to be enhanced to fulfill its needs.

Revised Manuscript Received on November 30, 2019.

* Correspondence Author

Panchagnula Sai Harish*, Department of Electrical \& Electronics Engineering, JNTUA College of Engineering, Ananthapuramu, India.

S.SRIDHAR, Assistant Professor, Department of Electrical\& Electronics Engineering, JNTUA College of Engineering, Ananthapuramu, India.

(C) The Authors. Published by Blue Eyes Intelligence Engineering and Sciences Publication (BEIESP). This is an open access article under the CC-BY-NC-ND license http://creativecommons.org/licenses/by-nc-nd/4.0/
A lot of control techniques are stepwise introduced to the BIM control system to enhance the performance of BIM control. The variable structure control technique in sliding mode belongs to modern controls, as the state itself can change frequently [3]. It posses excellent stability to, for

example, unsure limitations, system parameter disturbance or demand disruption, and inaccurate analytical designs. Eventually, the control system is accurately controlled and the SM variable system control method demonstrates the excellent usage opportunities in AC servo system control domain. In [4], a new disturbance observer is created to define the online rotational inertia scheme based on the linear switched SM controller, that weakens system steady status deviation and chattering aspect. Nevertheless, the status variable differentiation is brought into the surface of the dynamic SM, and the differential of velocity is required for speed control scheme, leading to high frequency noise, further reducing the controller's speed control efficiency. The system's mismatch and uncertain interferences are compensated by a nonlinear disturbance observer. It not only derives switching gain variation and maintains its nominal efficiency, but also improves the ability to reject system disruption. In [5], an enhanced sliding mode observer is designed on the principle of integral sliding surface and of differential sliding surface respectively for analysis of internal and external variations with load torque and rotation velocity as the target of understanding. A mixture of hybrid nonlinear feedback and integral SM methods is suggested and the synchronisation defects are adapted magnificently to null in the presence of the disturbances, suggested method achieves excellent solid output. A reaching law is suggested depending on the unique power model and sine of hyperbolic function, and the adjustable SM control law using the inverse hyperbolic sinusoidal function is also displayed, the suggested technique can efficiently decrease the input signal's high-frequency distortion. For an acquired reluctance motor, an essential SM control switch recurrence is displayed, and the sliding control unit's strength is analysed. However, the selection of parameter is difficult and the randomness of the framework plan on counter-hyperbolic sine job calculation is extended. Moreover, the choice of parameters is hard, and the difficulty of system development is increased on the basis of the inverse hyperbolic sinusoidal function model.

A specified switch rate integral sliding current control unit for a variable reluctance machine is displayed and the solidity of the sliding control is analyzed. 
Furthermore, the variable reluctance machine controller's parameter limit is obtained. This technique consists both standardized switching frequency and low frequency analysis. Moreover, there's still some impact on the velocity of the model without taking into account the wide variation under external disruption. In [6], DSMO is used respectively in poly phase bearing-less machine and pmsm, which is adaptable and robust to unsure and velocity interruptions. So sensorless control of superior performance is discovered. A tracking rule is suggested and implemented for discrete time SM control, efficiently enhancing scheme convergence velocity. A new adaptive universal SM control method consisting of a universal SM control framework and an adaptive tracker is proposed to achieve dominant locus finding efficiency regulation of unpredictable and unsure time changing structures.

Furthermore, the parameter requirement of the varying reluctance machine controller is determined. So the process consists a standardized recurrence of the switch just as inspection with low recurrence. In [8], a new flexible globally SM control approach based on a global SM control framework and a versatile tracker is implemented to gain predominance after execution control of unsafe and nonlinear time-fluctuating frameworks. Given the normal and exponential term of the primary request, a flexible variable-evaluated exponential methodology law is implemented that has the ability to adjust the sliding surface and the shift in the framework state. The presented method can normally regulate the velocity and weaken the chattering of the structure. Furthermore, on the assumption of the novel arriving at law, AESM control is suggested for BIM velocity control. In order to enhance the execution of sliding mode control against obstruction, an eyewitness aggravation SM is constructed, and its outcome is utilised as AESM control feed forward compensation.

\section{DESIGN OF AESM CONTROL}

Non-linear control model is taken as follows:

$$
\left\{\begin{array}{c}
x=f(t, x)+b(t, x)+g(t) \\
s=s(t, x)
\end{array}\right.
$$

where $x=\left[x_{1}, x_{2}\right]^{\mathbb{T}}$ are statue parameters; $b(t, x) \neq 0 ; g(t)$ denotes uncertain interference; $|g(t)| \leq \varsigma$, $\varsigma$ higher interference limit.

The SM has the qualities like pause and rigidity as a varaiable switch and makes the SM vibrations. Gao Wei Bing designs the traditional law index and it can be implemented as

$$
s=-\operatorname{ssgn}(s)-\lambda s \quad--(2)
$$

The arriving law can drain the system's internal vibrations to some extent and improve the speed adjustment control running value. It is, however, a vibration switch area close to the start, and the coefficient $\varepsilon, \lambda$ in the equation without self adjustment feature. Consequently, the optimum convergence feature cannot be accomplished. Depending on the status of the $\mathrm{s}$ and system, it is viable to propose a new adaptive variable rated exponential principle to overcome above difficulties:

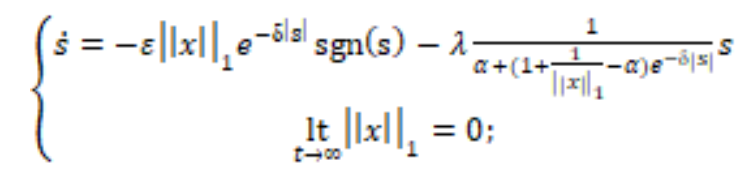

$$
\begin{aligned}
& \left\|x\left|\|_{1}=\sum_{i=1}^{n}\right| x \mid\right.
\end{aligned}
$$

is the order one standard of the system state parameter, $\lambda>0, \varepsilon>0,0<a<1, n>1, \delta>0$.

The scheme status variables in first order $\|x\|_{1}$ and the SM on-off capacity $|s|$ are presented by the arriving rule. The approach velocity is connected with distance from stability point between the state components and the sliding-mode switching component. Whenever ||$x \mid \|_{1}$ put far with $|s|$, where $|s|$ is high, the state parameter of framework deal with $|s|$ by methods for the list $-\lambda / \alpha$ s. Now, the non-linear term coefficient $\lambda / \alpha$ is higher compared to coefficient $\lambda$ of customary record arriving rule. In the meantime, the scheme alignment speed is enhanced by decreasing $\alpha$. As the state variable is equipped for racing to sliding surface, change in speed term $-\varepsilon\left\|x_{1}\right\| \operatorname{sgn}(s)$ assumes an important place. Simultaneously, the state falls in the SM surface and in order to reach steady point by activity of SM control action, $\|x\|_{1}$ diminishes continuously before reaching zero, and $-\varepsilon\left\|x_{1}\right\| \operatorname{sgn}(s)$ equals to zero. Vibration outcome of steady state speed term - $\operatorname{ssgn}(s)$ is debilitated and sliding balances out at origin at last.

A SM controller utilizing order one sliding surface is structured and is written as follows.

Axiom 1: Regarding non-linear control that is presented in equation (1), $s$ is written as:

$$
s=x_{1}+c_{0} \int_{0}^{t} x_{1} d t---(4)
$$

The controller can be designed as:

$$
u=b^{-1}(\mathrm{t}, \mathrm{x})\left[-\mathrm{f}(\mathrm{x})-\mathrm{g}(\mathrm{t})-\mathrm{e}\left\|x_{1}\right\| e^{-\delta|s|} \operatorname{sgn}(\mathrm{s})-\right.
$$

In a short time, the system can advance to a stable position, where $\mathrm{C}=\left[\begin{array}{ll}1 & c_{0}\end{array}\right]$

\section{Stability proof:}

The criteria for the development of specific sliding mode can be determined on the foundation of Lyapunov's principle of stability:

$$
V=\frac{1}{2} s^{2}<0
$$

Merging (3), and differentiating (6) with time, and making $1+\frac{1}{\|x\|_{4}}-a>0$. Thus $V$ can be denoted as

$$
\bar{V}=s\left[-\varepsilon \mid\left\|x_{1}\right\| e^{-5 \mid s]} \operatorname{sgn}(s)-\lambda \frac{1}{\alpha+\left(1+\frac{1}{\|x\|_{1}}-\alpha\right) e^{-\delta[s]}} s\right]
$$




$$
=-\varepsilon\left\|x_{1}\right\| e^{-\delta|s|}|s|-\lambda \frac{1}{\alpha+\left(1+\frac{1}{\|x \mid\|_{1}}-\alpha\right) e^{-\delta \mid s]}} s^{2}<0
$$

Hence, the AESMC approach fulfills the Lyapunov stability principle, and the system is assured to slide from the free spot in a definite time. Exponential sliding mode controller (SMC) and AESM control are constructed for standard nonlinear systems to confirm the superiority of the submitted technique.

$$
\left\{\begin{array}{c}
\dot{x}=B x+C u+D \\
s=A x
\end{array}\right.
$$

Where, $A=\left[\begin{array}{ll}2 & 12\end{array}\right], \quad B=\left[\begin{array}{cc}0 & 10 \\ 0 & 0\end{array}\right], \quad C=\left[\begin{array}{c}0 \\ -120\end{array}\right], \quad D=[1]$. Starting state parameters $\mathrm{X}(0)=[6$ 6], it is vividly observed in Fig.1 that AESM control have fast approaching speed, irregular oscillations are also suppressed.

\section{CONSTRUCTION OF BIM SPEED WITH AESM CONTROL}

\section{A. Modelling Of BIM}

The spiral suspension force based on electromagnetic field theory under the $d-q$ arrangement of the BIM is SHOWN as:

$$
\left\{\begin{array}{l}
F_{x}=K\left(i_{2 s d} \psi_{1 d}+i_{2 s q} \psi_{1 q}\right) \\
F_{y}=K\left(i_{2 s \sigma} \psi_{1 d}+i_{2 s d} \psi_{1 \sigma}\right)
\end{array}\right.
$$

Fx and Fy are segments of the outspread suspension force in $\mathrm{x}$ and $\mathrm{y}$ heading respectively; the subscript 1 and subscript 2 for the benefit of the torque turns and in the interest of levitation force turns respectively, $\mathrm{s}$ is for stator and $\mathrm{r}$ is for rotor, individually; $\psi_{-} 1 \mathrm{~d}$ and $\psi_{-}$1q are components of the torque winding transition linkage in the $\mathrm{d}-\mathrm{q}$ arrange, individually; i_2sd and i_2sq are components of stator current of suspension power twisting in $\mathrm{d}-\mathrm{q}$ arrange, separately; The electro magnetic torque condition is built up as:

The electromagnetic torque condition is built up as:

$$
\begin{gathered}
T_{e}=P_{1} \psi_{1} i_{q \varepsilon} \\
T_{e}-T_{1}=\frac{I}{P_{1}} \frac{d \omega}{d t}
\end{gathered}
$$

$\omega$ - rotor speed;

$i_{q s}$ - torque winding stator current in $q$ coordinate;

$T_{e}$ - electro magnetic torque;

$T_{1}$ - load torque;

$J$ - rotational inertia;

$P_{1}$ - torque winding pole logarithm.

\section{B. Design Of Velocity With AESM Control}

The state parameters are written as

$$
\left\{\begin{array}{c}
e_{w 1}=\omega^{*}-\omega \\
e_{w 2}=\int_{-\infty}^{t} e_{w 1} d t
\end{array}\right.
$$

where $\omega$ and $\omega^{*}$ are the actual and given velocity, respectively. According to the formulae of $(9 \sim 11), \dot{e}_{W \mathbb{1}}$ is given as:

$$
\dot{\theta}_{w 11}=-\dot{\omega}=-\frac{p_{1}^{2} \varphi_{1}}{I}+\frac{P_{1}}{I} T_{1}
$$

In case of variable change and external disturbances, $\mathscr{\omega}$ is given as:

$$
\dot{\omega}=\left(\frac{P_{1}^{2} \psi_{1}}{J}+\Delta \zeta\right) i_{q S}-\left(\frac{P_{1}}{J}+\Delta \eta\right) T_{1}+\Delta \xi
$$

where $\Delta \zeta, \Delta \eta$ and $\Delta \xi$ belongs to the uncertain interferences, $|\Delta \zeta| \leq v_{1}, \Delta \eta \leq v_{2}, \Delta \xi \leq v_{2} . v_{1}, v_{2}$ and $v_{3}$ are higher limit. Accordingly, $g_{1}(t)$ is noted as the final disruption, so, $\omega$ is reduced as:

$$
\dot{\omega}=\frac{P_{1}^{2} \Psi_{1}}{I} i_{q S}+g_{1}(t)
$$

where

$\theta_{1}(t)=(\Delta \zeta) i_{q s}+\left(\frac{P_{1}}{I}+\Delta \eta\right) T_{1}+\Delta \xi$ and

$\left|g_{1}(t)\right| \leq v, v$ is bounded positive integer. Thus, $\dot{\theta}_{\omega 1}$ is given as as:

$$
\dot{e}_{w 1}=-\frac{P_{1}^{2} \psi_{1}}{\mathbb{I}} i_{q S}-g_{1}(t)
$$

The velocity error equation is given as:

$$
\left\{\begin{array}{c}
\dot{e}_{\omega 1}=-\omega=-\frac{p_{1}^{2} \psi_{1}}{J} i_{q s}-g_{1}(t) \\
\dot{\theta}_{\omega 2}=e_{\omega 1}=\omega^{*}-\omega
\end{array}\right.
$$

When scheme arrives the sliding plane, $\mathrm{s}$ is given as:

$$
s=e_{w 1}+c \int_{0}^{t} e_{w 1} d t=0
$$

Differentiating the formula (17):

$$
e_{\text {WI }}=c_{0} e^{-t / c}
$$

From equation (18), it can be seen that the speed error become null depending on $e^{-1 / c}$, to attain the speed without over-shoot tracking. As a result, required SM motion can be attained by varying the $c$.

Incorporating equation (16), and deriving sliding surface function $s=e_{w 1}+c \int_{0}^{t} e_{w 1} d t$, s can be obtained as:

$$
s=\dot{\theta}_{w 1}+c e_{w 1}=-\frac{p_{1}^{2} \psi_{1}}{I} i_{q s}-g_{1}(t)+c \theta_{w 1}
$$

Combining the formula (3) and (19), it can be obtained as:

$$
-\varepsilon\left\|e_{\omega}\right\|_{1} e^{-\varepsilon|s|} \operatorname{sgn}(s)-\lambda \frac{1}{\alpha+\left(1+\frac{1}{\left\|e_{\omega}\right\|_{1}}-\alpha\right) e^{-\varepsilon \mid s} \mid} s=-\frac{P_{1}^{2} \psi_{1}}{J} i_{q s}-g_{1}(t)+c e_{\omega 1}
$$

From above equation, the $i_{q \varepsilon}$ can be designed as:

$$
\begin{aligned}
& i_{q s}=\frac{J}{p_{1}{ }^{2} \varphi_{1}}\left\{\varepsilon\left\|e_{\omega}\right\|_{1} e^{-\varepsilon \mid s]} \operatorname{sgn}(s)+\right. \\
& \left.\lambda \frac{1}{\alpha+\left[1+\frac{1}{\left\|\Phi_{w 1}\right\| I}-\alpha\right) e^{\left.\left.-S_{2}\right] x\right]}} s-g_{1}(t)+c e_{w 1}\right\}
\end{aligned}
$$

The speed controller AESM control of BIM can be noted as Axiom 2.

Axiom 2: Regarding the BIM rotating speed error controlling described in the equation (16), $s$ is written as:

$$
s=e_{w 11}+c \int_{0}^{t} e_{w 11} d t
$$

The controller $i_{q S}$ can be designed as:

$$
\begin{aligned}
& i_{q s}=\frac{J}{p_{\perp}^{2} \varphi_{1}}\left\{\varepsilon\left\|e_{\omega s}\right\|_{1} e^{\left.-\left.\delta\right|_{s}\right]} \operatorname{sgn}(s)+\right.
\end{aligned}
$$

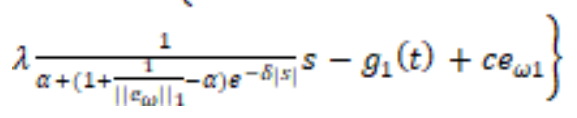


Thus, the speed error can converge in a definite time to required position. Variables for the scheme are $\lambda, \varepsilon, \omega$ and constant of integration $c$.

\section{MODELLING DSMO TO IDENTIFY DISRUPTION INERTIA}

\section{A. The construction and analysis of DSMO}

BIM's status equation can be given as:

$$
\left\{\begin{array}{c}
\omega=\frac{p_{1}^{2} \Psi_{1}}{J}+g_{1}(t) \\
g_{1}(t)=d(t)
\end{array}\right.
$$

where $d(t)$ denotes change of disturbance quantity $g_{1}(t)$ with respect to ' $\mathrm{t}$ '.

The preceding disturbance expansion SM observer is created on the basis of the above equation, sample objects with motor speed and system disturbance:

$$
\left\{\begin{array}{c}
\hat{\omega}=\frac{p_{1}^{2} \psi_{1}}{J} i_{q Q}+\hat{g}_{1}(t)+V \\
\hat{g}_{1}(t)=\vartheta V
\end{array}\right.
$$

where $V=\gamma \operatorname{sgn}(\hat{\omega}-\omega) ; \hat{\omega}$ is estimations of angular velocity and $\hat{g}_{1}(t)$ is system perturbation. $\gamma$ and $\vartheta$ are SM gain and feedback gain respectively;

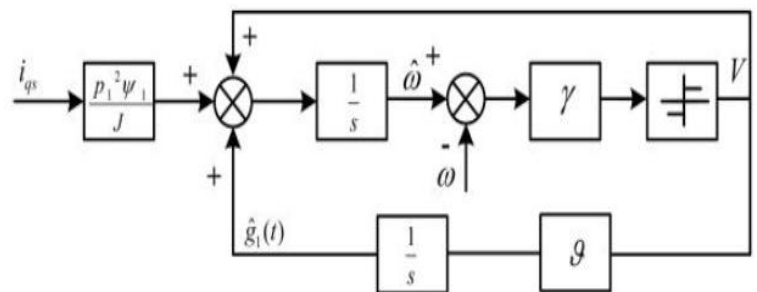

Figure 1: Schematic structure of system disturbance extended SM observer

Fig.1 represents an expanded SM observer schematic diagram, a velocity evaluation misstep and a disturbance evaluation error.:

$$
\left[\begin{array}{l}
x_{1} \\
x_{2}
\end{array}\right]=\left[\begin{array}{c}
\hat{\omega}-\omega \\
\hat{g}_{1}(t)-g_{1}(t)
\end{array}\right]
$$

Subtracting equation (24) from equation (25), the SM observation misstep is as follows:

$$
\left\{\begin{array}{c}
x_{1}^{0}=x_{2}+V \\
x_{2}^{n}=\vartheta V-d(t)
\end{array}\right.
$$

where $x_{1}$ denotes the speed and $x_{2}$ denotes misstep in estimating disturbance, $V$ gives switching function.

\section{B. Selection of variables for DSMO}

The parameters of observer need to be satisfied the condition of reaching of SM and the formula of observation misstep of SM (27) should be included in the (7). The SM's reaching condition may be described as:

$$
x_{1} \cdot x_{1}=x_{1}\left(x_{2}+V\right)=x_{1}\left[x_{2}+\gamma \operatorname{sgn}\left(x_{1}\right)\right]<0--(28)
$$

As a result, variable $\gamma$ needs to be satisfied as:

$$
\begin{gathered}
\gamma<\left|-x_{2}\right| \quad---(29) \\
\gamma=-m \mid x_{2} \|_{x} m>1 \quad---(30
\end{gathered}
$$

where $m$ denotes safety factor of SM.

$$
x_{1}=x_{1}^{x}=0
$$

Using above equation for the error formula (27):

$$
\left\{\begin{array}{c}
x_{2}=-V \\
x_{2}^{x}=\vartheta V-d(t)
\end{array}\right.
$$

This formula can be modified, and error of disturbance is obtained as:

$$
x_{2}=e^{-\theta t}\left[c+\int d(t) \cdot e^{\theta t} d t\right]--(33)
$$

where $c$ is constant.

To make error of estimation reach zero, the variable is considered as:

$$
\vartheta>0 \quad---(34)
$$

From equation (33), it is noted that convergence speed of the disruption estimation misstep $x_{2}$ is related by $\vartheta$.

Thus, the system ensures to converge to null in a definite moment by selecting variables in formula (30) and (34), and convergence speed can be modified.

\section{Study of DSMO chattering}

As DSMO ends gabbing, the chattering signal $\mathrm{C}$ is used to enhance finding exactness of the outside unsettling influence. Equation (32) can be reworked as:

$$
x_{2}+\vartheta x_{2}=\vartheta C-d(t)
$$

Consequently, the observation error transfer function, $x_{2}$ can be acquired as:

$$
F(s)=\frac{x_{2}}{c-T d[t]}=\frac{1}{T s+1} \quad---(36)
$$

where $F(s)$ is transfer function of low pass filter; $C$ and $T$ denotes chatter signal and the cycle rrespectively; $\omega_{c}=\frac{1}{\tau}=\vartheta$ represents the cut-off frequency.

From equation (36), it is noticed that low-pass filter filters out the chattering signal, which lowers the serious impacts of disruption on the system control.

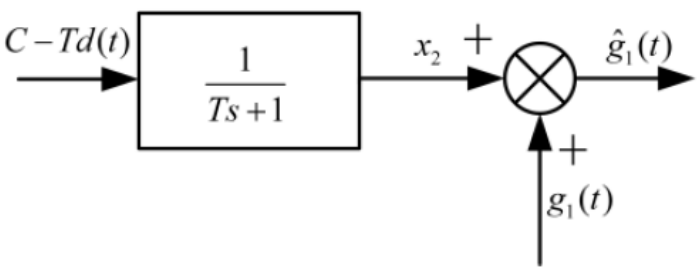

Figure 2: Schematic representation of the observer disturbance reduction 
The DSMO output is given to rectify input for system disruption with the feed forward compensation approach. The BIM velocity controller is therefore referred to as axiom 3 . Axiom 3: For the BIM speed deviation control displayed in equation (16), $\mathrm{s}$ is chosen as follows:

$$
s=e_{w 1}+c \int_{0}^{t} e_{w 1} d t
$$

The given current Iqs can be available as:

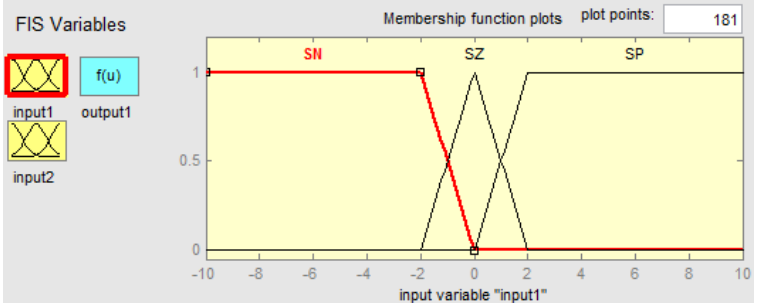

Figure 4: Input 1 Membership Functions

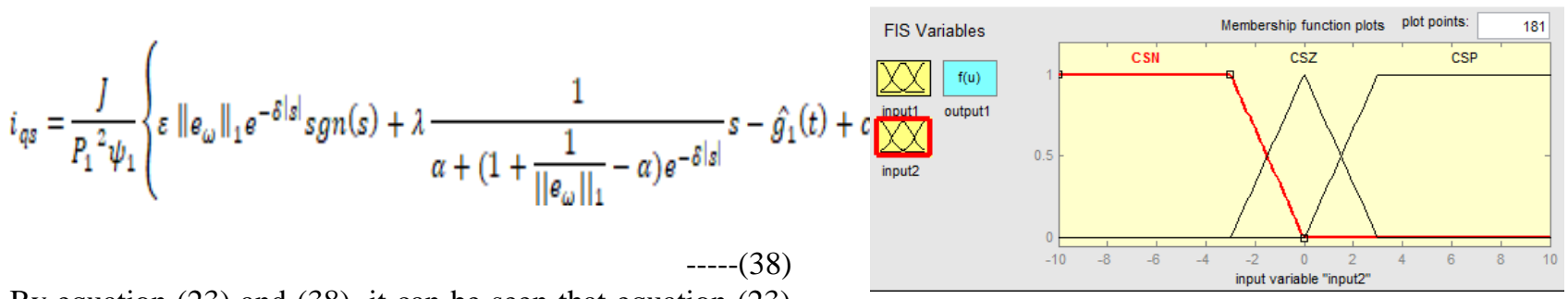

By equation (23) and (38), it can be seen that equation (23) that variables $\varepsilon$ and $\mathrm{k}$ must be high in order to meet the adjustment in load disruption at expense of raising the magnitude of irregular quantity and buffeting. But, the observed amount of disruption feedback to the given current in equation (38) can still provide present requirement without the need for big $\varepsilon$ and $\mathrm{k}$

The system disruption is therefore modified in real time and at the same time the disruption free stability is enhanced.

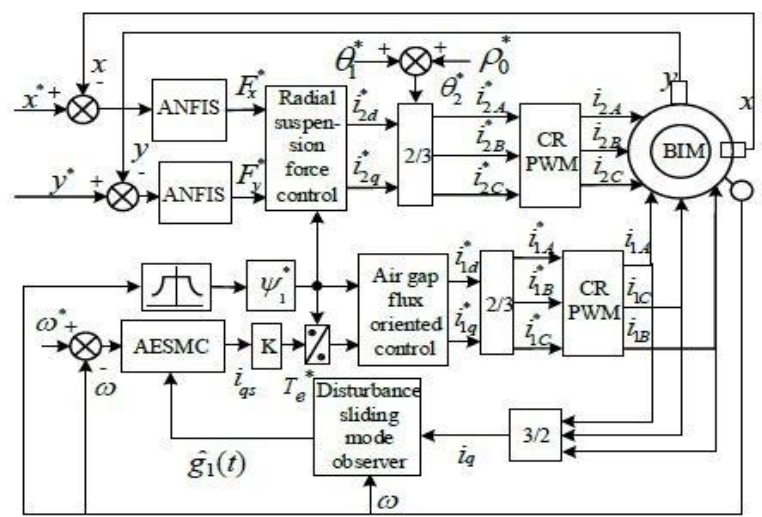

Figure 3 : Block diagram of BIM sliding-mode control

\section{CONTROLLING USING ADAPTIVE NEURO FUZZY INFERENCE SYSTEM (ANFIS)}

ANFIS is a kind of artificial neural network that is based on Takagi-Sugeno fuzzy inference system. It is the combination of both fuzzy logic principles and neural networks, it has strength to get the advantages of both in a single framework. Its inference process refers to a group of fuzzy IF-THEN conditions that have learning capability to approximate non-linear functions. Thus, ANFIS is considered to be a universal estimator. For utilizing the ANFIS in a progressively effective and ideal way, one can utilize the best parameters acquired by genetic calculation. Below figures show the ANFIS controller functions and structures of the proposed system.

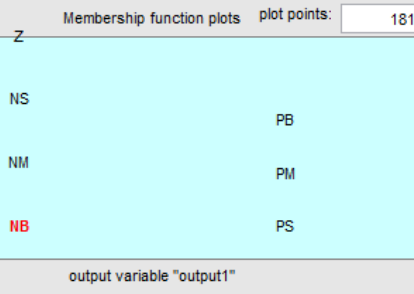

Figure 6: Output Membership Functions

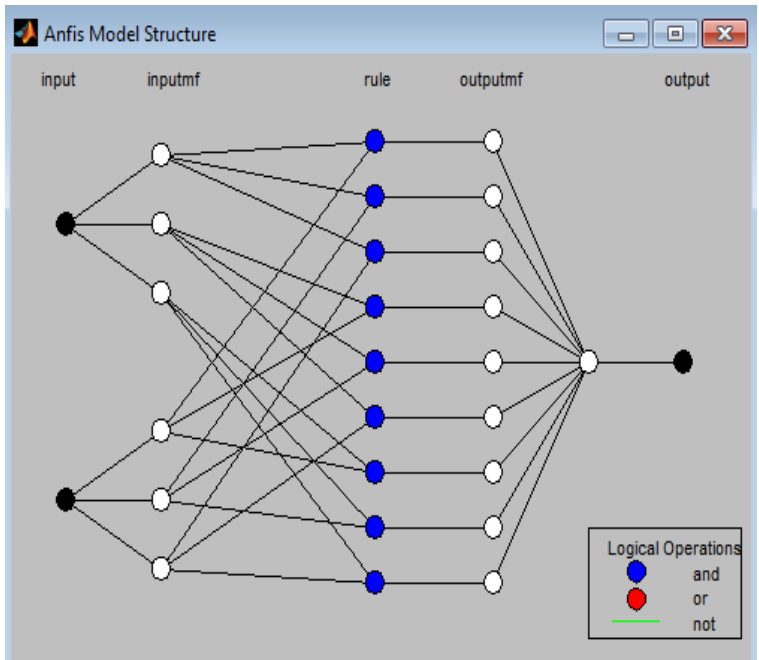

Figure 7: ANFIS model Structure

\section{SIMULATION RESULTS}

A. Simulation results using SMC and AESM control

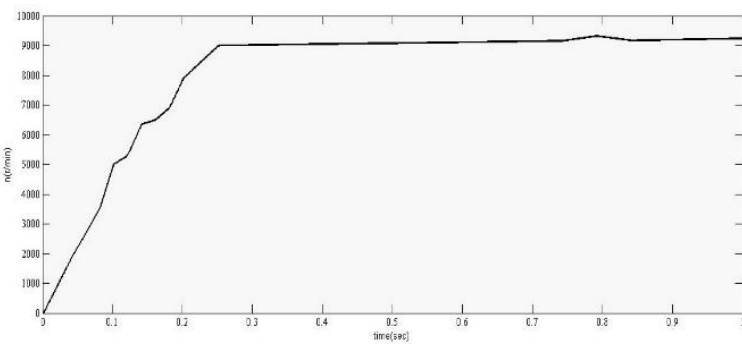

IJITEE$$
\text { iences Publication }
$$ 


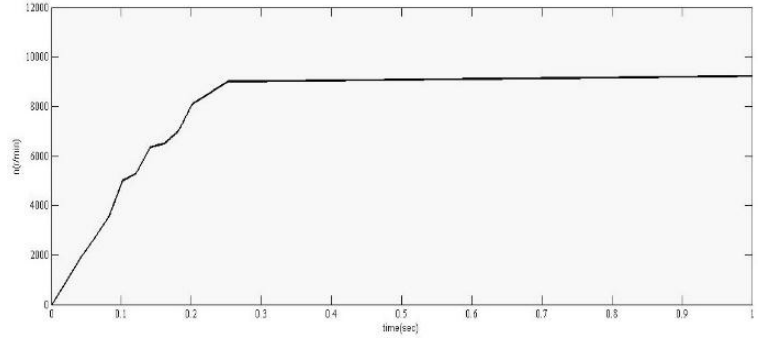

Figure 8: Velocity waveforms of load mutation
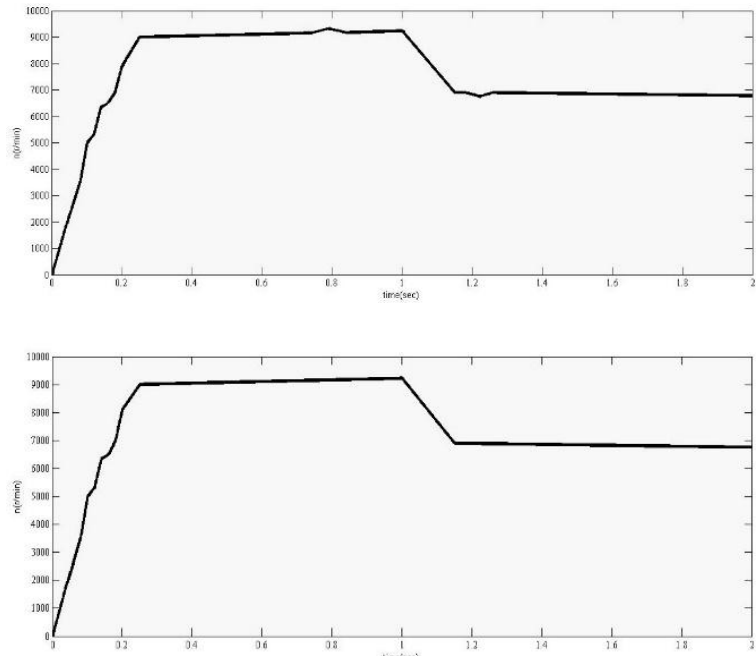

Figure 9: Velocity waveforms under the torque perturbation

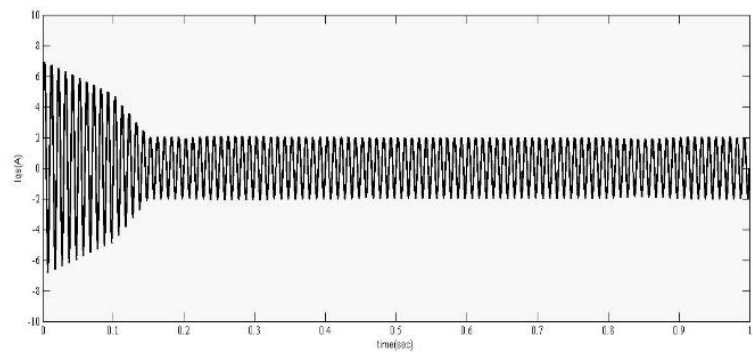

Figure 10: Current response waveforms

B. Simulation results using Adaptive Neuro Fuzzy Inference System:

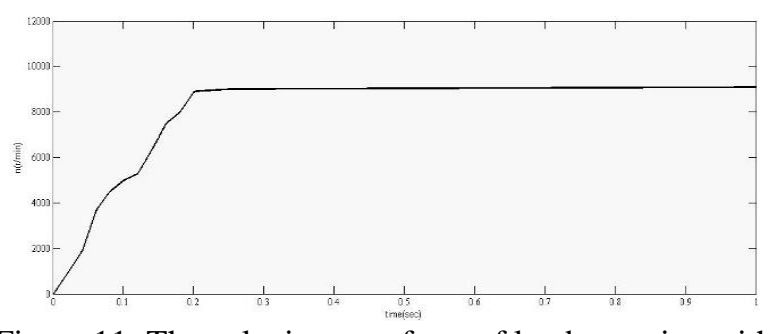

Figure 11: The velocity waveform of load mutation with ANFIS
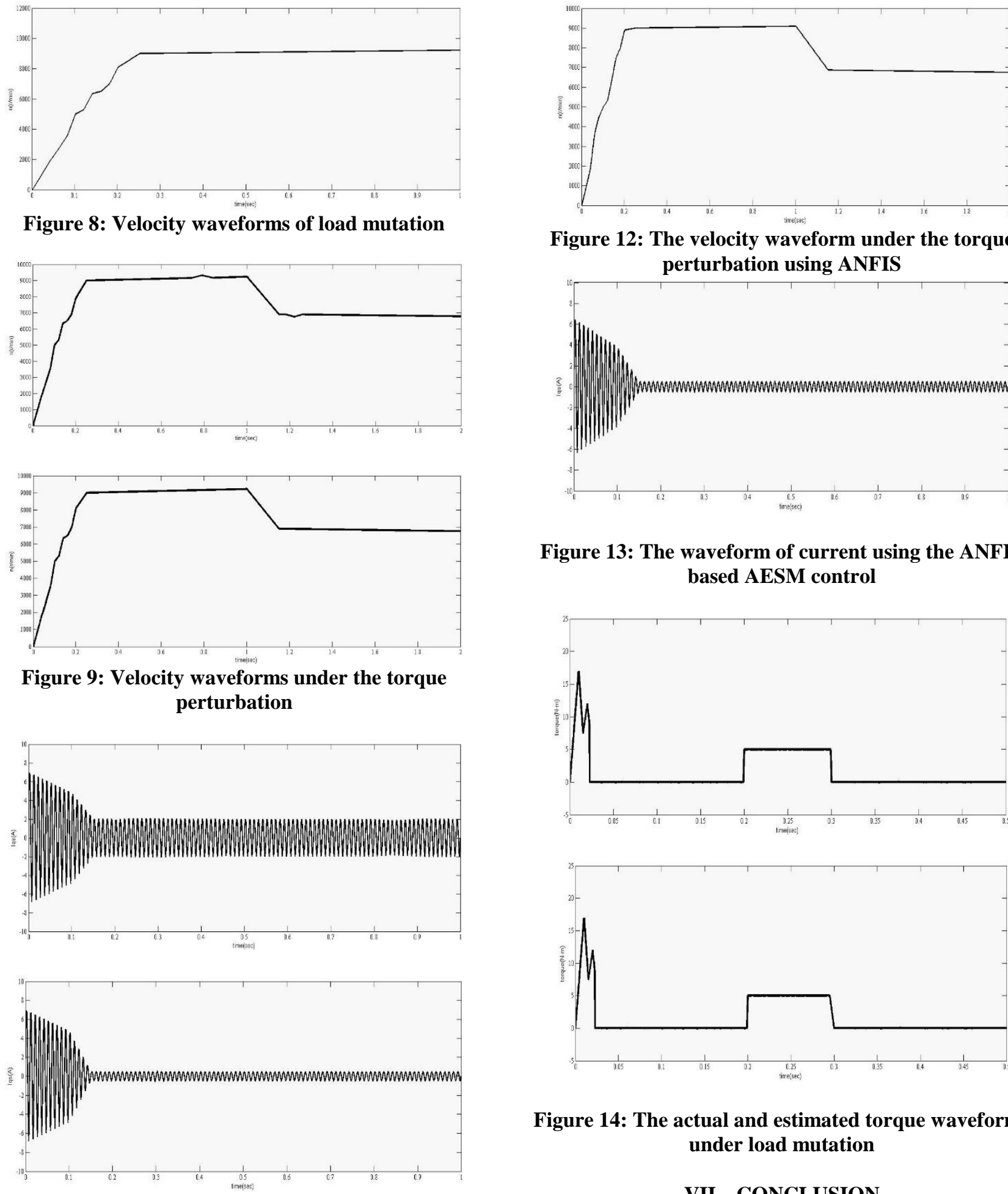

Figure 12: The velocity waveform under the torque perturbation using ANFIS

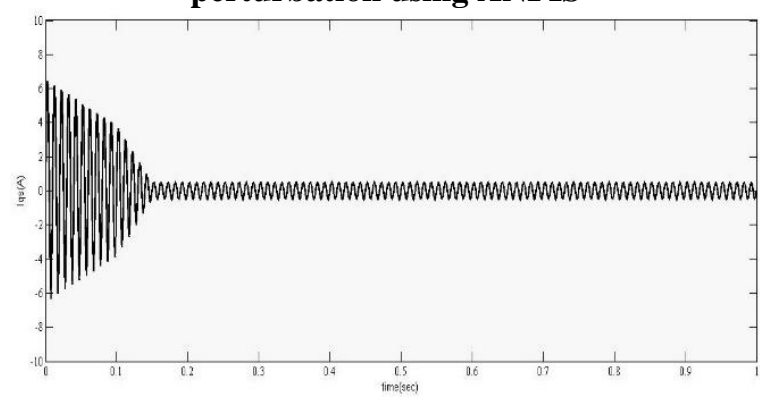

Figure 13: The waveform of current using the ANFIS based AESM control
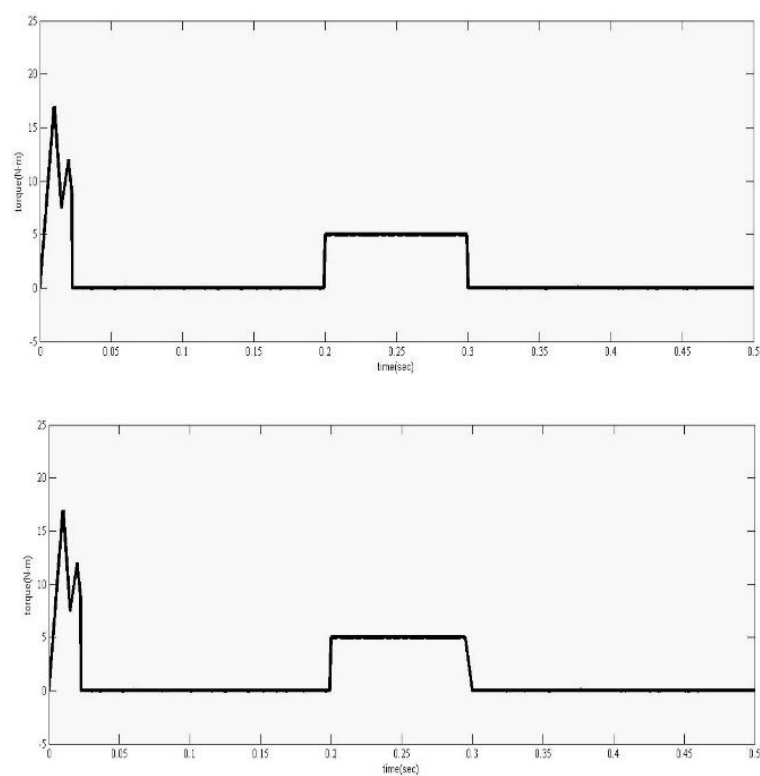

Figure 14: The actual and estimated torque waveforms under load mutation

\section{CONCLUSION}

An advanced DSMO based on the AESM control is suggested to attain the capacity of large progressive response and good interference resistance for the BIM drive scheme. The suggested approach can modify the arriving velocity of the status parameter distance from equilibrium position automatically and suppress the system chatter. Furthermore, expanded DSMO can assess the system disruption in real time and the outcome is utilised to compensate disturbance that decreases discontinuous magnitude and increases the system's capacity to anti-interfere. Instead of standard PI controller, we used an ANFIS based controller.

Published By: 
The simulation findings indicate that the suggested control approach with ANFIS controller not only demonstrates that the level of interference has no impact on the control system efficiency, but also efficiently increases the dynamic output of BIM and the capacity to prevent interference. The suggested strategy can be seen as a successful approach to managing the AC servo system. Further research on levitation force winding control can be conducted to rapidly and precisely track the specified radial displacement value using the AESM control with ANFIS based controller.

\section{REFERENCES}

1. Sun X, Chen L, Yang Z. Overview of bearingless permanent magnet synchronous motors[J]. IEEE Transactions on Industrial Electronics, 2013.

2. SINERVO A, ARKKIO A. Rotor radial position control and its effect on the total efficiency of a bearingless induction motor with a cage rotor[J]. IEEE Transactions on Magnetics, 2014.

3. Mobayen S. Chaos synchronization of uncertain chaotic systems using composite nonlinear feedback based integral sliding mode control[J]. ISA Transactions, 2018.

4. Liu Y, Zhou B, Fang S. Sliding mode control of PMSM based on a novel disturbance observer[J]. Proceedings of the CSEE, 2010.

5. Zhang X, Sun L, Zhao K. Sliding mode control of PMSM based on a novel load torque sliding mode observer[J]. Proceedings of the CSEE, 2012.

6. Lu W, Lin H, Han J. Position Sensorless Control of Permanent Magnet Synchronous Machine Using a Disturbance Observer[J]. Proceedings of the CSEE, 2016.

\section{AUTHORS FROFILE}

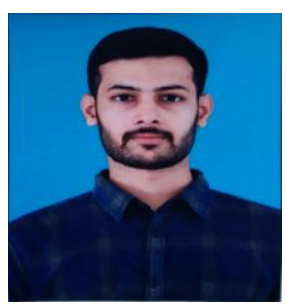

Panchagnula Sai Harish, received his Bachelor's Degree in Electrical \& Electronics Engineering from Kuppam Engineering College affiliated to Jawaharlal Nehrru Technological University, Ananathapuram, INDIA. .He is currently working towards his Master's degree in Power \& Industrial Drives specialization from Department of Electrical \& Electronics Engineering, JNTUA College of Engineering, Ananthapuramu, India. His research interests are Electrical Drives and new control schemes related to drives.

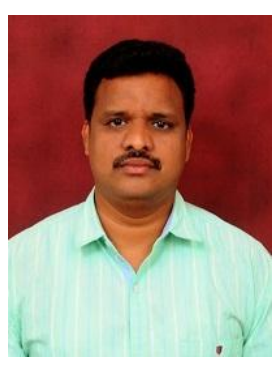

Ananthapuramu, India.
S.SRIDHAR, received B.Tech in Electrical \& Electronics Engineering in 2003 from JNTU Hyderabad. He received M.Tech in Electrical Machines \& Industrial Drives in 2006 from JNTU Hyderabad. He is working towards his Ph.D. He has teaching experience of 8 years. He published 25 International journals, 3 National Conferences, 3 International conferences. Guided 50 post graduate projects and 8 under graduate projects. He is working as Assistant professor in Electrical Department, JNTUA College of Engineering, 